\title{
Codes and analog codes in chemistry
}

\author{
Lutvo Kurić \\ Institute of Economics, University of Sarajevo, Trg Oslobođenja 1, Sarajevo, \\ Bosnia and Herzegovina \\ E-mail address: lutvokuric@yahoo.com
}

\begin{abstract}
This paper discusses cyberinformation studies of the chemical elements composition in nature, in particular the identification of scientific terminology that could describe this phenomenon, ie, the study of biochemical information, as well as the relationship between the digital language of chemical elements and theoretical aspects of this elements and cybernetics. In digital pictures of biochemistry, physical and chemical parameters are in a strict submission to programmed, cyber and information rules. In some examples, chemical elements are connected through the discrete code 931 , which is transformed into $3^{2} 3^{1} 3^{0}$ code.
\end{abstract}

Keywords: Digital chemistry; chemical code; analog code; digital language of chemistry

\section{INTRODUCTION}

The biochemical role of biochemistry depends on the positioning of its component chemical elements, and is understood by the ,positioning of elements forming words“. Each of these words has its biochemical base. If this base is expressed by corresponding discrete numbers, it can be seen that any given base has its own program, along with its own unique cybernetics and information characteristics.

Indeed, the sequencing of the biochemistry is determined not only by distin biochemical features, but also by cybernetic and information principles. For this reason, research in this field deals more with the quantitative rather than qualitative characteristcs of biochemical information and its biochemical basis. For the purposes of this paper, specific physical and chemical factors have been selected in order to express the chemical information for chemical elements. Numerical values are them assigned to these factors, enabling them to be measured. In this way it is possible to determine oif a connection really exists between the quantitative ratios in the process of transfer of biochemical information and the qualitative appearance of the biochemistry. To select these factors, preference is given to classical physical and chemical parameters, including the atomic numbers in the relevant chemical elements, their analog values, the position in periodic table, and their frenquencies. There is a arge numbers of these parameters, and each of their gives important biochemical information.

Going through these parameters, it becomes clear that there is a mathematical relationship between quantitative ratios and the qualitative appearance of the chemical elements and that there is a measurement method that can be used to describe the biochemistry of those elements.. 


\section{RESULTS}

The matrix mechanism of $\mathrm{c}$ hemistry, the evolution of groups and periods and, especially, the biochemical evolution of periodic table language, have been analyzed by the application of cybernetic methods, information theory and system theory, respectively. The primary structure of a periodic table is the exact specification of its atomic number composition and the chemical bonds connecting those numbers.

Codes and analog codes in chemistry we can find using the following algorithm:

$$
\begin{gathered}
\{[(\mathrm{SB}(\mathrm{X} 1,2,3, \mathrm{n}) \times \mathrm{A})]-[(\mathrm{SA}(\mathrm{X} 1,2,3, \mathrm{n}) \times \mathrm{B})]\}+(\mathrm{AB})\}=\mathrm{ABA} \\
\mathrm{A}=19 ; \mathrm{B}=7 ; \\
\{[(\mathrm{S} 7(\mathrm{X} 1,2,3, \mathrm{n}) \times \mathrm{A})]-[(\mathrm{S} 19(\mathrm{X} 1,2,3, \mathrm{n}) \times \mathrm{B})]\}+(\mathbf{A B})\}=(\mathbf{1 9 \times 7 \times 1 9}) ; \\
(\mathbf{1 9} \mathbf{7} \mathbf{7} \mathbf{1 9})=\mathbf{9 3 1} ; \\
\mathbf{9 3 1}>\mathbf{9} \mathbf{3} \mathbf{1} \mathbf{3}^{\mathbf{2}} \mathbf{3}^{\mathbf{1}} \mathbf{3}^{\mathbf{0}} ; \\
\mathrm{S}=\text { Groups of atomic numbers } 1,2,3, \mathrm{n} \\
\mathrm{X} 1,2,3, \mathrm{n}=\text { Atomic numbers } 1,2,3, \mathrm{n}
\end{gathered}
$$

Discrete code $\mathbf{3}^{\mathbf{2}} \mathbf{3}^{\mathbf{1}} \mathbf{3}^{\mathbf{0}}$ inter connects some chemical elements. That connection is established through various parameters. These are: odd and even values, primary and secondary values, positioning of chemical elements in a given group of elements, etc.

\section{Group of chemical elements}

\begin{tabular}{|c|c|c|c|c|}
\hline $\mathbf{8}$ & $\mathbf{9}$ & $\mathbf{1 5}$ & $\mathbf{2 4}$ & $\mathbf{2 5}$ \\
$\underline{\mathrm{O}}$ & $\underline{\mathrm{F}}$ & $\underline{\mathrm{P}}$ & $\underline{\mathrm{Cr}}$ & $\underline{\mathrm{Mn}}$ \\
\hline $\mathbf{2 6}$ & $\mathbf{3 0}$ & $\mathbf{3 6}$ & $\mathbf{4 3}$ & $\mathbf{4 4}$ \\
$\underline{\mathrm{Fe}}$ & $\underline{\mathrm{Zn}}$ & $\underline{\mathrm{Kr}}$ & $\underline{\mathrm{Tc}}$ & $\underline{\mathrm{Ru}}$ \\
\hline 45 & $\mathbf{4 6}$ & $\mathbf{4 7}$ & $\underline{49}$ & $\mathbf{5 9}$ \\
$\underline{\mathrm{Rh}}$ & $\underline{\mathrm{Pd}}$ & $\underline{\mathrm{Ag}}$ & $\underline{\mathrm{In}}$ & $\underline{\mathrm{Pr}}$ \\
\hline $\mathbf{6 0}$ & $\mathbf{6 1}$ & $\mathbf{6 2}$ & $\mathbf{6 3}$ & $\mathbf{6 4}$ \\
$\underline{\mathrm{Nd}}$ & $\underline{\mathrm{Pm}}$ & $\underline{\mathrm{Sm}}$ & $\underline{\mathrm{Eu}}$ & $\underline{\mathrm{Gd}}$ \\
\hline $\mathbf{6 5}$ & $\mathbf{7 9}$ & $\mathbf{8 7}$ & $\mathbf{8 8}$ & $\mathbf{9 3}$ \\
$\underline{\mathrm{Tb}}$ & $\underline{\mathrm{Au}}$ & $\underline{\mathrm{Fr}}$ & $\underline{\mathrm{Ra}}$ & $\underline{\mathrm{Np}}$ \\
\hline
\end{tabular}

Fig. 1. Group of chemical elements in the Chemical Elements Table

In this table we have a group of 25 chemical elements. These elements are interconnected through the discrete code $\mathbf{3}^{\mathbf{2}} \mathbf{3}^{\mathbf{1}} \mathbf{3}^{\mathbf{0}}$. This code connects them in the following way: 


\section{Inner and outer atomic numbers}

\begin{tabular}{|c|c|c|c|c|}
\hline 8 & 9 & 15 & 24 & 25 \\
$\underline{0}$ & $\underline{F}$ & $\underline{P}$ & $\underline{\mathrm{Cr}}$ & $\underline{\mathrm{Mn}}$ \\
\hline 26 & 30 & 36 & 43 & 44 \\
$\underline{\mathrm{Fe}}$ & $\underline{\mathrm{Zn}}$ & $\underline{\mathrm{Kr}}$ & $\underline{\mathrm{Tc}}$ & $\underline{\mathrm{Ru}}$ \\
\hline 45 & 46 & 47 & 49 & 59 \\
$\underline{\mathrm{Rh}}$ & $\underline{\mathrm{Pd}}$ & $\underline{\mathrm{Ag}}$ & $\underline{\mathrm{In}}$ & $\underline{\mathrm{Pr}}$ \\
\hline 60 & 61 & 62 & 63 & 64 \\
$\underline{\mathrm{Nd}}$ & $\underline{\mathrm{Pm}}$ & $\underline{\mathrm{Sm}}$ & $\underline{\mathrm{Eu}}$ & $\underline{\mathrm{Gd}}$ \\
\hline 65 & 79 & 87 & 88 & 93 \\
$\mathrm{~Tb}$ & $\mathrm{Au}$ & $\mathrm{Fr}$ & $\mathrm{Ra}$ & $\mathrm{Np}$ \\
\hline
\end{tabular}

Fig. 2. The blue shading is for the outer, and green for inner atomic numbers of the group of chemical elements in question.

Inner atomic numbers $=(30+36+43+46+47+49+61+62+63)=437$;

Outer atomic numbers

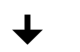

$(8+9+15+24+25+26+44+45+59+60+64+65+79+87+88+93)=791$;

Inner code $=437$; Analog inner code $=734$;

734|| 437

Outer code $=791 ;$ Analog outer code $=197$;

$197 \| 791$

$(734+197)=$ Discret code $931>\mathbf{3}^{\mathbf{2}} \mathbf{3}^{\mathbf{1}} \mathbf{3}^{\mathbf{0}}$.

\section{Notes:}

Each atomic number has its own analogue form. That form is one of constants in the programmed, cyber and information systems in biochemistry.

The analogue code together with the code is yet another proof that the process of sequencing in the biomacromolecules is really conditioned and determined, not only by biochemical, but also cyber-information rules.

In this example the atomic numbers of the group of chemical elements in question are interconnected through the discrete code $3^{\mathbf{2}} \mathbf{3}^{\mathbf{1}} \mathbf{3}^{\mathbf{0}}$.

This connection is not visible one, so we do not know if it exists. This connection can be established using inner and outer atomic numbers and their analogue codes, which can clearly be observed in the previous example. 


\section{Comment:}

Interpreting as: ListCorrelate $[\{8,9,15,24,25,26,44,45,49,59,60,64,65,79,87,88,93\}$, $\{30,36,43,46,47,49,61,62,63\}^{\wedge}$ Conjugate $\left.[\mathrm{x}],\{1\}\right]$

\section{Input:}

ListCorrelate $[\{8,9,15,24,25,26,44,45,49,59,60,64,65,79,87,88,93\}$, $\left.\{30,36,43,46,47,49,61,62,63\}^{x^{*}},\{1\}\right]$

\section{Result:}

$$
\begin{aligned}
& \left\{5 \times 3^{2 x^{*}+1} 4^{x^{*}+1}+7^{x^{*}+2} 9^{x^{*}}+4^{x^{*}} 9^{x^{*}+1}+2^{x^{*}+3} 15^{x^{*}}+\right. \\
& 3 \times 2^{x^{*}+3} 23^{x^{*}}+59 \times 30^{x^{*}}+3 \times 2^{x^{*}} 31^{x^{*}+1}+79 \times 43^{x^{*}}+ \\
& 65 \times 46^{x^{*}}+104 \times 47^{x^{*}}+113 \times 49^{x^{*}}+132 \times 61^{x^{*}}+45 \times 62^{x^{*}} \text {, } \\
& 31 \times 3^{2 x^{*}+1} 7^{x^{*}}+2^{2 x^{*}+3} 9^{x^{*}}+5 \times 7^{x^{*}} 9^{x^{*}+1}+2^{x^{*}+6} 23^{x^{*}}+ \\
& 49 \times 30^{x^{*}}+11 \times 2^{x^{*}+2} 31^{x^{*}}+11 \times 2^{x^{*}+3} 31^{x^{*}}+59 \times 36^{x^{*}}+ \\
& 69 \times 43^{x^{*}}+15 \times 46^{x^{*}}+89 \times 47^{x^{*}}+104 \times 49^{x^{*}}+113 \times 61^{x^{*}} \text {, } \\
& 2^{x^{*}} 3^{x^{*}+2} 5^{x^{*}+1}+44 \times 3^{2 x^{*}+1} 7^{x^{*}}+31 \times 3^{x^{*}+1} 10^{x^{*}}+15 \times 2^{x^{*}+2} 23^{x^{*}}+13 \times 2^{x^{*}+1} 31^{x^{*}}+ \\
& 49 \times 36^{x^{*}}+67 \times 43^{x^{*}}+9 \times 46^{x^{*}}+79 \times 47^{x^{*}}+89 \times 49^{x^{*}}+104 \times 61^{x^{*}}+87 \times 62^{x^{*}} \text {, } \\
& 31 \times 3^{2 x^{*}+1} 4^{x^{*}}+29 \times 3^{2 x^{*}+1} 7^{x^{*}}+5 \times 4^{x^{*}} 9^{x^{*}+1}+11 \times 2^{x^{*}+2} 15^{x^{*}}+ \\
& 11 \times 2^{x^{*}+3} 15^{x^{*}}+2^{x^{*}+3} 23^{x^{*}}+13 \times 2^{x^{*}+3} 31^{x^{*}}+49 \times 43^{x^{*}}+ \\
& 59 \times 46^{x^{*}}+69 \times 47^{x^{*}}+79 \times 49^{x^{*}}+89 \times 61^{x^{*}}+26 \times 63^{x^{*}} \text {, } \\
& 11 \times 2^{2 x^{*}+3} 9^{x^{*}}+11 \times 4^{x^{*}+1} 9^{x^{*}}+29 \times 3^{x^{*}+1} 10^{x^{*}}+13 \times 2^{x^{*}+1} 15^{x^{*}}+3 \times 2^{x^{*}+3} 31^{x^{*}}+ \\
& 138 \times 43^{x^{*}}+49 \times 46^{x^{*}}+67 \times 47^{x^{*}}+69 \times 49^{x^{*}}+79 \times 61^{x^{*}}+65 \times 62^{x^{*}}+104 \times 63^{x^{*}} \text {, } \\
& 29 \times 3^{2 x^{*}+1} 4^{x^{*}}+5^{x^{*}+2} 6^{x^{*}}+8 \times 3^{2 x^{*}+1} 7^{x^{*}}+13 \times 2^{2 x^{*}+1} 9^{x^{*}}+79 \times 30^{x^{*}}+2^{x^{*}+6} 31^{x^{*}}+ \\
& 132 \times 43^{x^{*}}+3 \times 46^{x^{*}+1}+49 \times 47^{x^{*}}+67 \times 49^{x^{*}}+69 \times 61^{x^{*}}+15 \times 62^{x^{*}}+65 \times 63^{x^{*}} \text {, } \\
& 2^{x^{*}+3} 3^{x^{*}+1} 5^{x^{*}}+13 \times 5^{x^{*}+1} 6^{x^{*}}+5 \times 3^{2 x^{*}+1} 7^{x^{*}}+13 \times 2^{2 x^{*}+3} 9^{x^{*}}+ \\
& 11 \times 2^{x^{*}+2} 23^{x^{*}}+11 \times 2^{x^{*}+3} 23^{x^{*}}+15 \times 2^{x^{*}+2} 31^{x^{*}}+113 \times 43^{x^{*}}+ \\
& 138 \times 47^{x^{*}}+49^{x^{*}+1}+67 \times 61^{x^{*}}+9 \times 62^{x^{*}}+64 \times 63^{x^{*}} \text {, } \\
& 2^{2 x^{*}+3} 3^{2 x^{*}+1}+20 \times 3^{2 x^{*}+1} 7^{x^{*}}+7^{x^{*}} 9^{x^{*}+1}+2^{x^{*}+6} 15^{x^{*}}+2^{x^{*}} 15^{x^{*}+1}+ \\
& 13 \times 2^{x^{*}+1} 23^{x^{*}}+2^{x^{*}+3} 31^{x^{*}}+65 \times 36^{x^{*}}+104 \times 43^{x^{*}}+ \\
& 87 \times 46^{x^{*}}+132 \times 47^{x^{*}}+138 \times 49^{x^{*}}+49 \times 61^{x^{*}}+59 \times 62^{x^{*}} \text {, } \\
& 5 \times 3^{2 x^{*}+1} 4^{x^{*}}+4^{x^{*}+3} 9^{x^{*}}+3^{x^{*}+2} 10^{x^{*}}+2^{x^{*}+2} 15^{x^{*}+1}+13 \times 2^{x^{*}+3} 23^{x^{*}}+ \\
& \left.89 \times 43^{x^{*}}+113 \times 47^{x^{*}}+132 \times 49^{x^{*}}+138 \times 61^{x^{*}}+49 \times 62^{x^{*}}+67 \times 63^{x^{*}}\right\}
\end{aligned}
$$




\section{Plots:}

( $x$ from -2 to 1 )

$-5 \times 3^{2 x^{*}+1} 4^{x^{*}+1}+7^{x^{*}+2} 9^{x^{*}}+4^{x^{*}} 9^{x^{*}+1}+2^{x^{*}+3} 15^{x^{*}}+$ $3 \times 2^{x^{*}+3} 23^{x^{*}}+59 \times 30^{x^{*}}+3 \times 2^{x^{*}} 31^{x^{*}+1}+79 \times 43^{x^{*}}+$ $65 \times 46^{x^{*}}+104 \times 47^{x^{*}}+113 \times 49^{x^{*}}+132 \times 61^{x^{*}}+45 \times$ $62^{x}$

$-31 \times 3^{2 x^{*}+1} 7^{*}+2^{2 x^{*}+3} 9^{*}+5 \times 7^{*} 9^{*}+1+2^{*}+6$ $23^{x^{*}}+49 \times 30^{*}+11 \times 2^{x^{*}+2} 31^{*}+11 \times 2^{*}+331^{*}+$ $59 \times 36^{x^{*}}+69 \times 43^{x^{*}}+15 \times 46^{x^{*}}+89 \times 47^{x^{*}}+104 \times 49^{x^{*}}+$ $113 \times 61^{x}$

$-2^{x^{*}} 3^{x^{*}+2} 5^{x^{*}+1}+44 \times 3^{2 x^{*}+1} 7^{x^{*}}+31 \times 3^{x^{*}+1} 10^{x^{*}}+15 \times$ $2^{x^{*}+2} 23^{x^{*}}+13 \times 2^{x^{*}+1} 31^{x^{*}}+49 \times 36^{x^{*}}+67 \times 43^{x^{*}}+9 \times$ $46^{x^{*}}+79 \times 47^{x^{*}}+89 \times 49^{x^{*}}+104 \times 61^{x^{*}}+87 \times 62^{x^{*}}$

$-31 \times 3^{2 x^{*}+1} 4^{x^{*}}+29 \times 3^{2 x^{*}+1} 7^{*}+5 \times 4^{*} 9^{*}+1+11 \times$ $2^{x^{*}+2} 15^{x^{*}}+11 \times 2^{x^{*}+3} 15^{x^{*}}+2^{x^{*}+3} 23^{x^{*}}+13 \times 2^{x^{*}+3}$

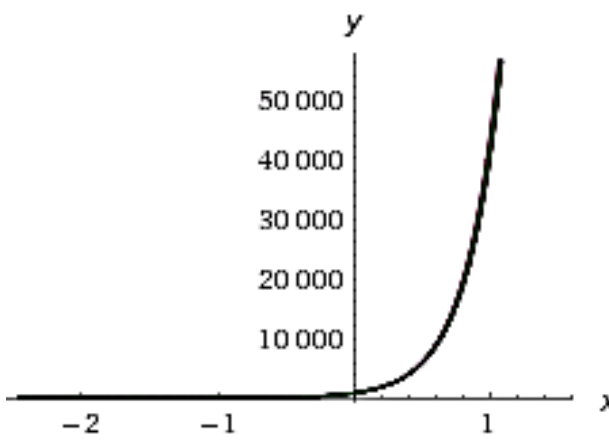
$31^{x^{*}}+49 \times 43^{x^{*}}+59 \times 46^{x^{*}}+69 \times 47^{x^{*}}+79 \times 49^{x^{*}}+89 \times$ $61^{x^{*}}+26 \times 63^{x}$

$-11 \times 2^{2 x^{*}+3} 9^{x^{*}}+11 \times 4^{x^{*}+1} 9^{*}+29 \times 3^{*}+110^{*}+13 \times$ $2^{x^{*}+1} 15^{x^{*}}+3 \times 2^{x^{*}+3} 31^{x^{*}}+138 \times 43^{x^{*}}+49 \times 46^{x^{*}}+67 \times$ $47^{x^{*}}+69 \times 49^{x^{*}}+79 \times 61^{x^{*}}+65 \times 62^{x^{*}}+104 \times 63^{x^{*}}$

$-29 \times 3^{2 x^{*}+1} 4^{x^{*}}+5^{x^{*}+2} 6^{x^{*}}+8 \times 3^{2 x^{*}+1} 7^{*}+13 \times$ $2^{2 x^{*}+1} 9^{x^{*}}+79 \times 30^{x^{*}}+2^{x^{*}+6} 31^{x^{*}}+132 \times 43^{x^{*}}+3 \times$ $46^{x^{*}+1}+49 \times 47^{x^{*}}+67 \times 49^{x^{*}}+69 \times 61^{x^{*}}+15 \times 62^{x^{*}}+$ $65 \times 63^{x}$

$-2^{x^{*}+3} 3^{x^{*}+1} 5^{x^{*}}+13 \times 5^{x^{*}+1} 6^{x^{*}}+5 \times 3^{2 x^{*}+1} 7^{*}+13 \times$ $2^{2 x^{*}+3} 9^{x^{*}}+11 \times 2^{x^{*}+2} 23^{x^{*}}+11 \times 2^{x^{*}+3} 23^{x^{*}}+15 \times$ $2^{x^{*}+2} 31^{x^{*}}+113 \times 43^{x^{*}}+138 \times 47^{x^{*}}+49^{x^{*}+1}+67 \times$ $61^{x^{*}}+9 \times 62^{x^{*}}+64 \times 63^{x^{*}}$

$-2^{2 x^{*}+3} 3^{2 x^{*}+1}+20 \times 3^{2 x^{*}+1} 7^{\dot{x}}+7^{*} 9^{*}+1+2^{\dot{x}+6}$ $15^{x^{*}}+2^{x^{*}} 15^{x^{*}+1}+13 \times 2^{x^{*}+1} 23^{x^{*}}+2^{x^{*}+3} 31^{x^{*}}+65 x$ $36^{x^{*}}+104 \times 43^{x^{*}}+87 \times 46^{x^{*}}+132 \times 47^{x^{*}}+138 \times 49^{x^{*}}+$ $49 \times 61^{x}+59 \times 62^{x}$

$-5 \times 3^{2 x^{*}+1} 4^{x^{*}}+4^{x^{*}+3} 9^{x^{*}}+3^{x^{*}+2} 10^{x^{*}}+2^{x^{*}+2} 15^{x^{*}+1}+$ $13 \times 2^{x^{*}+3} 23^{x^{*}}+89 \times 43^{x^{*}}+113 \times 47^{x^{*}}+132 \times 49^{x^{*}}+$ $138 \times 61^{x^{*}}+49 \times 62^{x^{*}}+67 \times 63^{x^{*}}$ 


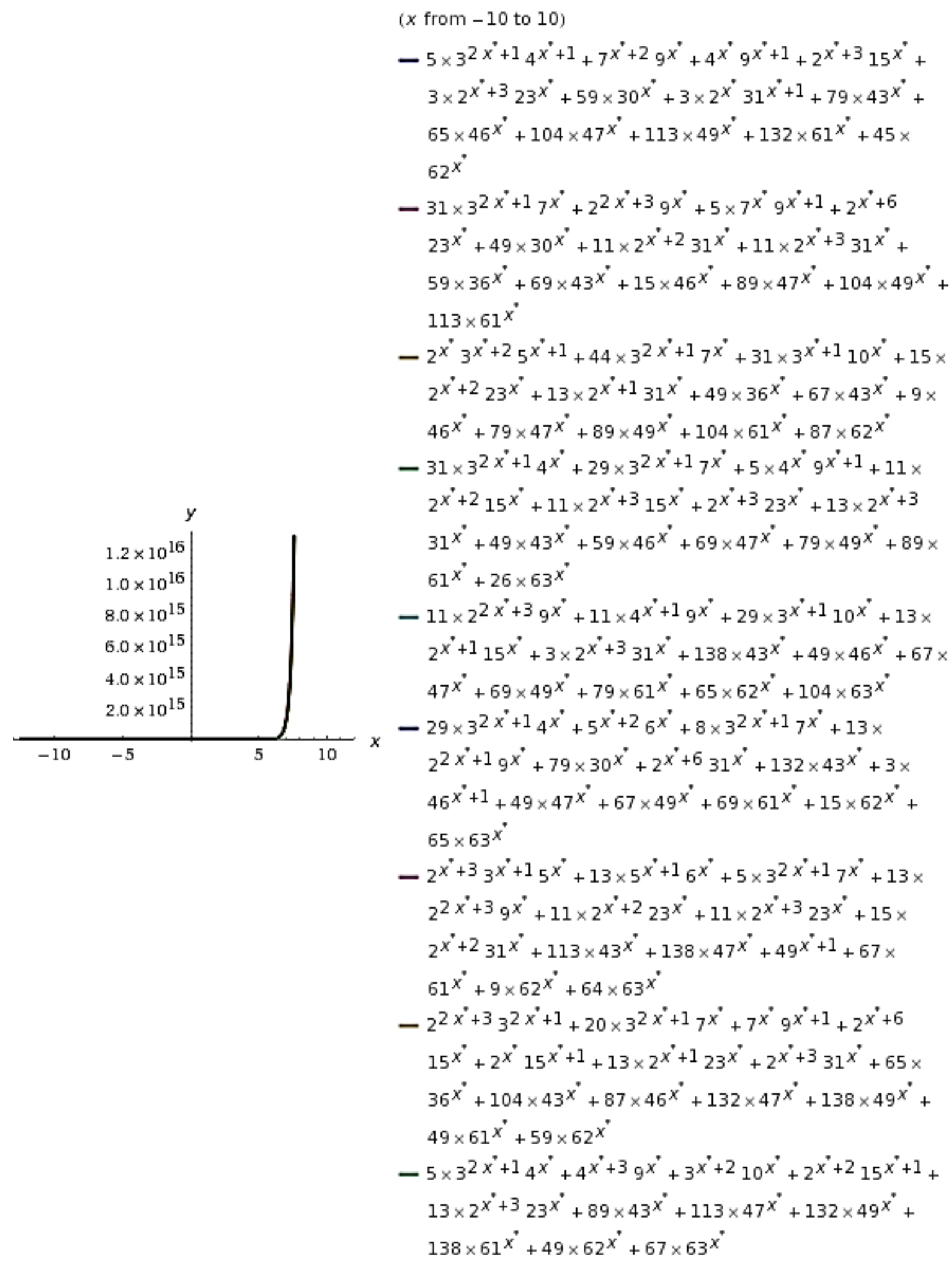




\section{Outer and inner numbers}

\begin{tabular}{|c|c|c|c|c|}
\hline 8 & 9 & 15 & 24 & 25 \\
$\underline{O}$ & $\underline{F}$ & $\underline{P}$ & $\underline{\mathrm{Cr}}$ & $\mathrm{Mn}$ \\
\hline 26 & 30 & $\mathbf{3 6}$ & 43 & 44 \\
$\underline{\mathrm{e}}$ & $\underline{\mathrm{Zn}}$ & $\underline{\mathrm{Kr}}$ & $\underline{\mathrm{Tc}}$ & $\underline{\mathrm{Ru}}$ \\
\hline 45 & 46 & 47 & 49 & 59 \\
$\mathrm{Rh}$ & $\underline{\mathrm{Pd}}$ & $\underline{\mathrm{Ag}}$ & $\underline{\mathrm{In}}$ & $\underline{\mathrm{Pr}}$ \\
\hline 60 & $\mathbf{6 1}$ & $\mathbf{6 2}$ & $\mathbf{6 3}$ & $\mathbf{6 4}$ \\
$\mathrm{Nd}$ & $\underline{\mathrm{Pm}}$ & $\underline{\mathrm{Sm}}$ & $\underline{\mathrm{Eu}}$ & $\underline{\mathrm{Gd}}$ \\
\hline 65 & 79 & $\mathbf{8 7}$ & $\mathbf{8 8}$ & 93 \\
$\underline{\mathrm{Tb}}$ & $\underline{\mathrm{Au}}$ & $\underline{\mathrm{Fr}}$ & $\underline{\mathrm{Ra}}$ & $\underline{\mathrm{Np}}$ \\
\hline
\end{tabular}

Outer numbers $=(8+9+15+24+25+26+44+45+59+60+64+65+79+8788+93)=791$;

Analog code $=197$

Inner numbers $=(30+36+43+46+47+49+61+62+63)=437$;

Analog code $=734$

$$
(\mathrm{A} 9+\mathrm{A} 10)=(197+734)=\mathbf{9 3 1}
$$

\section{Comment:}

Interpreting as: ListCorrelate $[\{8,9,15,24,25,26,44,45,59,60,64,65,79,87,88,93\},\{30,36$, $43,46,47,49,61,62,63\}^{\wedge}$ Conjugate[x], \{1\}]

\section{Input:}

ListCorrelate $[\{8,9,15,24,25,26,44,45,59,60,64,65,79,87,88,93\}$, $\left.\{30,36,43,46,47,49,61,62,63\}^{x^{*}},\{1\}\right]$ 


\section{Result:}

$$
\begin{aligned}
& \left\{4^{x^{*}+3} 9^{x^{*}}+4^{x^{*}} 9^{x^{*}+1}+2^{x^{*}+3} 15^{x^{*}}+2^{x^{*}+2} 15^{x^{*}+1}+3 \times 2^{x^{*}+3} 23^{x^{*}}+80 \times 43^{x^{*}}+\right. \\
& 79 \times 46^{x^{*}}+112 \times 47^{x^{*}}+114 \times 49^{x^{*}}+137 \times 61^{x^{*}}+45 \times 62^{x^{*}}+59 \times 63^{x^{*}} \text {, } \\
& 5 \times 3^{2 x^{*}+1} 4^{x^{*}+1}+16 \times 7^{2 x^{*}+1}+2^{2 x^{*}+3} 9^{x^{*}}+5 \times 7^{x^{*}} 9^{x^{*}+1}+5 \times 2^{x^{*}+4} 23^{x^{*}}+ \\
& 59 \times 30^{x^{*}}+11 \times 2^{x^{*}+2} 31^{x^{*}}+3 \times 2^{x^{*}} 31^{x^{*}+1}+73 \times 43^{x^{*}}+103 \times 47^{x^{*}}+114 \times 61^{x^{*}} \text {, } \\
& 2^{x^{*}} 3^{x^{*}+2} 5^{x^{*}+1}+31 \times 3^{2 x^{*}+1} 7^{x^{*}}+2^{x^{*}+6} 23^{x^{*}}+13 \times 2^{x^{*}+1} 31^{x^{*}}+11 \times 2^{x^{*}+3} 31^{x^{*}}+ \\
& 59 \times 36^{x^{*}}+68 \times 43^{x^{*}}+9 \times 46^{x^{*}}+80 \times 47^{x^{*}}+103 \times 49^{x^{*}}+112 \times 61^{x^{*}}+44 \times 63^{x^{*}} \text {, } \\
& 38 \times 3^{2 x^{*}+1} 7^{x^{*}}+5 \times 4^{x^{*}} 9^{x^{*}+1}+31 \times 3^{x^{*}+1} 10^{x^{*}}+11 \times 2^{x^{*}+2} 15^{x^{*}}+15 \times 2^{x^{*}+2} 23^{x^{*}}+ \\
& 2^{x^{*}+3} 23^{x^{*}}+7 \times 2^{x^{*}+4} 31^{x^{*}}+59 \times 43^{x^{*}}+73 \times 47^{x^{*}}+80 \times 49^{x^{*}}+103 \times 61^{x^{*}} \text {, } \\
& 31 \times 3^{2 x^{*}+1} 4^{x^{*}}+29 \times 3^{2 x^{*}+1} 7^{x^{*}}+11 \times 4^{x^{*}+1} 9^{x^{*}}+13 \times 2^{x^{*}+1} 15^{x^{*}}+ \\
& 11 \times 2^{x^{*}+3} 15^{x^{*}}+3 \times 2^{x^{*}+3} 31^{x^{*}}+45 \times 43^{x^{*}}+59 \times 46^{x^{*}}+ \\
& 68 \times 47^{x^{*}}+73 \times 49^{x^{*}}+80 \times 61^{x^{*}}+79 \times 62^{x^{*}}+25 \times 63^{x^{*}} \text {, } \\
& 5^{x^{*}+2} 6^{x^{*}}+8 \times 3^{2 x^{*}+1} 7^{x^{*}}+13 \times 2^{2 x^{*}+1} 9^{x^{*}}+11 \times 2^{2 x^{*}+3} 9^{x^{*}}+29 \times 3^{x^{*}+1} 10^{x^{*}}+ \\
& 5 \times 2^{x^{*}+4} 31^{x^{*}}+137 \times 43^{x^{*}}+45 \times 46^{x^{*}}+59 \times 47^{x^{*}}+68 \times 49^{x^{*}}+73 \times 61^{x^{*}}+79 \times 63^{x^{*}} \text {, } \\
& 29 \times 3^{2 x^{*}+1} 4^{x^{*}}+2^{x^{*}+3} 3^{x^{*}+1} 5^{x^{*}}+5 \times 3^{2 x^{*}+1} 7^{x^{*}}+11 \times 2^{x^{*}+2} 23^{x^{*}}+ \\
& 79 \times 30^{x^{*}}+2^{x^{*}+6} 31^{x^{*}}+25 \times 36^{x^{*}}+114 \times 43^{x^{*}}+93 \times 46^{x^{*}}+ \\
& 45 \times 47^{x^{*}}+59 \times 49^{x^{*}}+68 \times 61^{x^{*}}+9 \times 62^{x^{*}}+65 \times 63^{x^{*}} \text {, } \\
& 2^{2 x^{*}+3} 3^{2 x^{*}+1}+13 \times 5^{x^{*}+1} 6^{x^{*}}+7^{x^{*}} 9^{x^{*}+1}+2^{x^{*}} 15^{x^{*}+1}+13 \times 2^{x^{*}+1} 23^{x^{*}}+ \\
& 11 \times 2^{x^{*}+3} 23^{x^{*}}+15 \times 2^{x^{*}+2} 31^{x^{*}}+2^{x^{*}+3} 31^{x^{*}}+79 \times 36^{x^{*}}+ \\
& 112 \times 43^{x^{*}}+137 \times 47^{x^{*}}+45 \times 49^{x^{*}}+59 \times 61^{x^{*}}+64 \times 63^{x^{*}} \text {, } \\
& 5 \times 3^{2 x^{*}+1} 4^{x^{*}}+20 \times 3^{2 x^{*}+1} 7^{x^{*}}+3^{x^{*}+2} 10^{x^{*}}+2^{x^{*}+6} 15^{x^{*}}+7 \times 2^{x^{*}+4} 23^{x^{*}}+ \\
& \left.65 \times 36^{x^{*}}+103 \times 43^{x^{*}}+114 \times 47^{x^{*}}+137 \times 49^{x^{*}}+45 \times 61^{x^{*}}+59 \times 62^{x^{*}}+8 \times 63^{x^{*}}\right\}
\end{aligned}
$$




\section{Plots:}

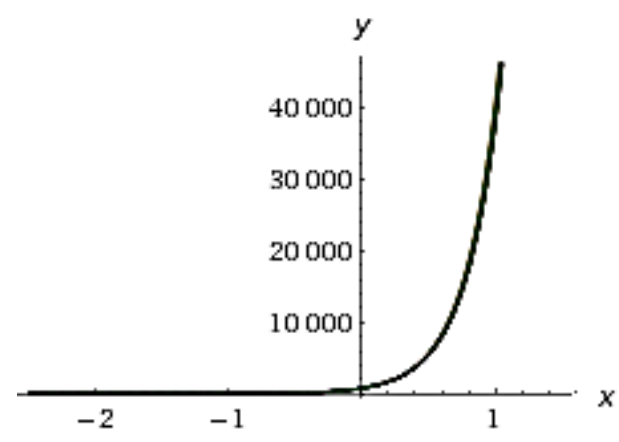

( $x$ from -2 to 1 )

$-4^{x^{*}+3} 9^{x^{*}}+4^{x^{*}} 9^{x^{*}+1}+2^{x^{*}+3} 15^{x^{*}}+2^{x^{*}+2} 15^{x^{*}+1}+3 x$ $2^{x^{*}+3} 23^{x^{*}}+80 \times 43^{x^{*}}+79 \times 46^{x^{*}}+112 \times 47^{x^{*}}+114 x$ $49^{x^{*}}+137 \times 61^{x^{*}}+45 \times 62^{x^{*}}+59 \times 63^{x^{*}}$

$-5 \times 3^{2 x^{*}+1} 4^{x^{*}+1}+16 \times 7^{2 x^{*}+1}+2^{2 x^{*}+3} 9^{x^{*}}+5 \times 7^{x^{*}}$ $9^{x^{*}+1}+5 \times 2^{x^{*}+4} 23^{x^{*}}+59 \times 30^{x^{*}}+11 \times 2^{x^{*}+2} 31^{x^{*}}+3 \times$ $2^{x^{*}} 31^{x^{*}+1}+73 \times 43^{x^{*}}+103 \times 47^{x^{*}}+114 \times 61^{x^{*}}$ $-2^{x^{*}} 3^{x^{*}+2} 5^{x^{*}+1}+31 \times 3^{2 x^{*}+1} 7^{x^{*}}+2^{x^{*}+6} 23^{x^{*}}+13 x$ $2^{x^{*}+1} 31^{x^{*}}+11 \times 2^{x^{*}+3} 31^{x^{*}}+59 \times 36^{x^{*}}+68 \times 43^{x^{*}}+9 \times$ $46^{x^{*}}+80 \times 47^{x^{*}}+103 \times 49^{x^{*}}+112 \times 61^{x^{*}}+44 \times 63^{x^{*}}$

$-38 \times 3^{2 x^{*}+1} 7^{x^{*}}+5 \times 4^{x^{*}} 9^{x^{*}+1}+31 \times 3^{x^{*}+1} 10^{x^{*}}+11 \times$ $2^{x^{*}+2} 15^{x^{*}}+15 \times 2^{x^{*}+2} 23^{x^{*}}+2^{x^{*}+3} 23^{x^{*}}+7 \times 2^{x^{*}+4}$ $31^{x^{*}}+59 \times 43^{x^{*}}+73 \times 47^{x^{*}}+80 \times 49^{x^{*}}+103 \times 61^{x^{*}}$ $-31 \times 3^{2 x^{*}+1} 4^{x^{*}}+29 \times 3^{2 x^{*}+1} 7^{x^{*}}+11 \times 4^{x^{*}+1} 9^{x^{*}}+13 \times$ $2^{x^{*}+1} 15^{x^{*}}+11 \times 2^{x^{*}+3} 15^{x^{*}}+3 \times 2^{x^{*}+3} 31^{x^{*}}+45 x$ $43^{x^{*}}+59 \times 46^{x^{*}}+68 \times 47^{x^{*}}+73 \times 49^{x^{*}}+80 \times 61^{x^{*}}+79 \times$ $62^{x^{*}}+25 \times 63^{x^{*}}$

$-5^{x^{*}+2} 6^{x^{*}}+8 \times 3^{2 x^{*}+1} 7^{x^{*}}+13 \times 2^{2 x^{*}+1} 9^{x^{*}}+11 \times$ $2^{2 x^{*}+3} 9^{x^{*}}+29 \times 3^{x^{*}+1} 10^{x^{*}}+5 \times 2^{x^{*}+4} 31^{x^{*}}+137 x$ $43^{x^{*}}+45 \times 46^{x^{*}}+59 \times 47^{x^{*}}+68 \times 49^{x^{*}}+73 \times 61^{x^{*}}+79 \times$ $63^{x}$

$-29 \times 3^{2 x^{*}+1} 4^{x^{*}}+2^{x^{*}+3} 3^{x^{*}+1} 5^{x^{*}}+5 \times 3^{2 x^{*}+1} 7^{x^{*}}+11 \times$ $2^{x^{*}+2} 23^{x^{*}}+79 \times 30^{x^{*}}+2^{x^{*}+6} 31^{x^{*}}+25 \times 36^{x^{*}}+114 x$ $43^{x^{*}}+93 \times 46^{x^{*}}+45 \times 47^{x^{*}}+59 \times 49^{x^{*}}+68 \times 61^{x^{*}}+9 \times$ $62^{x^{*}}+65 \times 63^{x^{*}}$

$-2^{2 x^{*}+3} 3^{2 x^{*}+1}+13 \times 5^{x^{*}+1} 6^{x^{*}}+7^{x^{*}} 9^{x^{*}+1}+2^{x^{*}} 15^{x^{*}+1}+$ $13 \times 2^{x^{*}+1} 23^{x^{*}}+11 \times 2^{x^{*}+3} 23^{x^{*}}+15 \times 2^{x^{*}+2} 31^{x^{*}}+$ $2^{x^{*}+3} 31^{x^{*}}+79 \times 36^{x^{*}}+112 \times 43^{x^{*}}+137 \times 47^{x^{*}}+45 \times$ $49^{x^{*}}+59 \times 61^{x^{*}}+64 \times 63^{x^{*}}$

$-5 \times 3^{2 x^{*}+1} 4^{x^{*}}+20 \times 3^{2 x^{*}+1} 7^{x^{*}}+3^{x^{*}+2} 10^{x^{*}}+2^{x^{*}+6}$ $15^{x^{*}}+7 \times 2^{x^{*}+4} 23^{x^{*}}+65 \times 36^{x^{*}}+103 \times 43^{x^{*}}+114 \times$ $47^{x^{*}}+137 \times 49^{x^{*}}+45 \times 61^{x^{*}}+59 \times 62^{x^{*}}+8 \times 63^{x^{*}}$ 


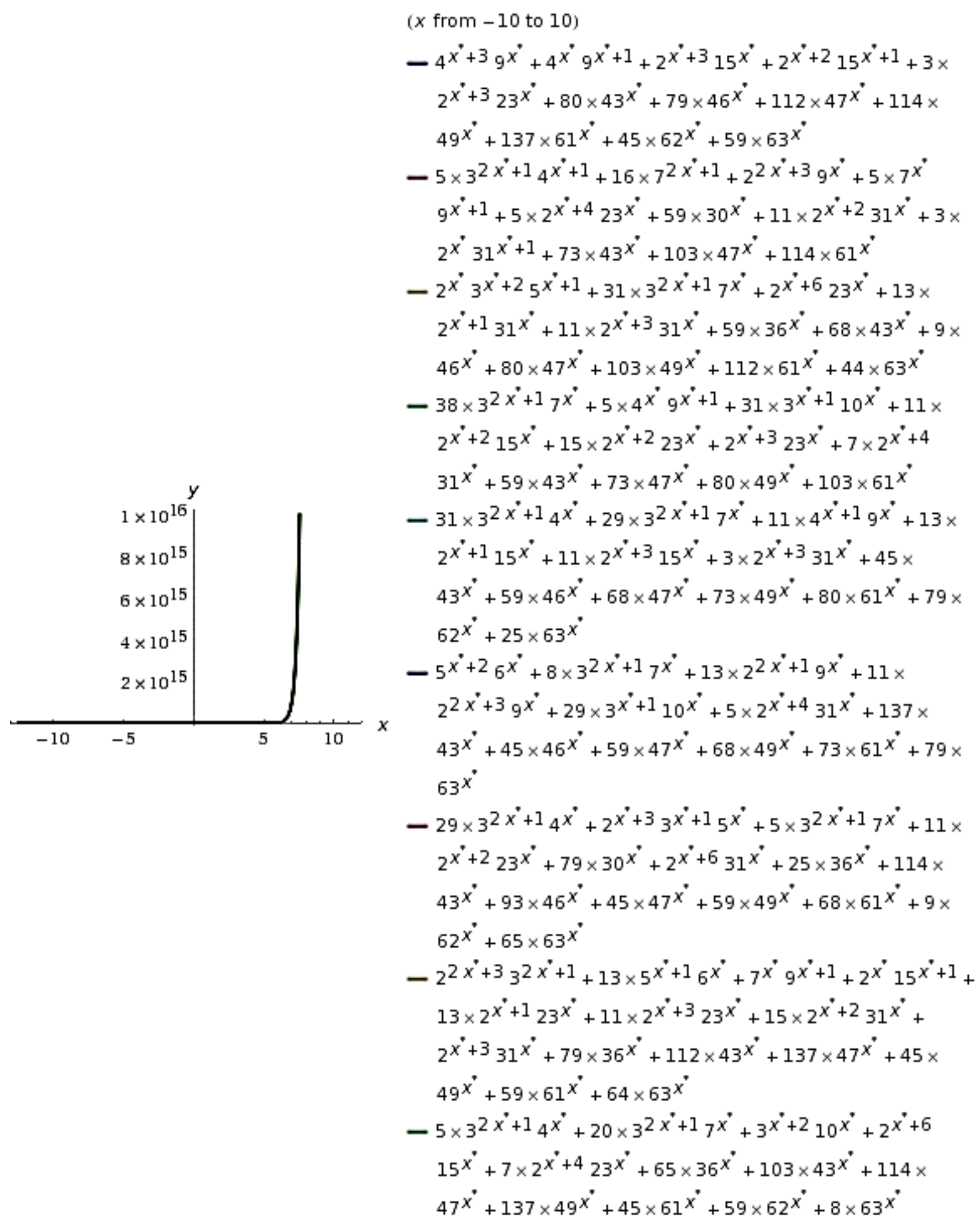




\section{Even and odd atomic numbers}

\begin{tabular}{|c|c|c|c|c|}
\hline 8 & 9 & 15 & 24 & 25 \\
$\underline{0}$ & $\underline{F}$ & $\underline{P}$ & $\underline{\mathrm{Cr}}$ & $\underline{\mathrm{Mn}}$ \\
\hline 26 & 30 & 36 & 43 & 44 \\
$\underline{\mathrm{Fe}}$ & $\underline{\mathrm{Zn}}$ & $\underline{\mathrm{Kr}}$ & $\underline{\mathrm{Tc}}$ & $\underline{\mathrm{Ru}}$ \\
\hline 45 & 46 & 47 & 49 & 59 \\
$\mathrm{Rh}$ & $\mathrm{Pd}$ & $\mathrm{Ag}$ & $\mathrm{In}$ & $\mathrm{Pr}$ \\
\hline 60 & 61 & 62 & 63 & 64 \\
$\mathrm{Nd}$ & $\mathrm{Pm}$ & $\mathrm{Sm}$ & $\underline{\mathrm{Eu}}$ & $\mathrm{Gd}$ \\
\hline 65 & 79 & 87 & 88 & 93 \\
$\underline{\mathrm{Tb}}$ & $\underline{\mathrm{Au}}$ & $\underline{\mathrm{Fr}}$ & $\underline{\mathrm{Ra}}$ & $\underline{\mathrm{Np}}$ \\
\hline
\end{tabular}

The blue shading is for the even, and green for odd atomic numbers of the group of chemical elements in question.

Even atomic numbers $=488 ;$ Odd atomic numbers $=740$;

Even code $=488 ;$ Analog even code $=884$;

Odd code $=740 ;$ Analog odd code $=047$

$$
(884+47)=\text { Discret code } 931>\mathbf{3}^{\mathbf{2}} \mathbf{3}^{\mathbf{1}} \mathbf{3}^{\mathbf{0}} .
$$

In this example, the chemical elements are interconnected through even and odd atomic numbers and their analogue values. The connection between them is the discrete code $\mathbf{9 3 1}$.

\section{Even and odd rank}

\begin{tabular}{|c|c|c|c|c|}
\hline 8 & 9 & 15 & 24 & 25 \\
o & $\mathrm{F}$ & $\mathrm{P}$ & $\mathrm{Cr}$ & $\mathrm{Mn}$ \\
\hline 26 & 30 & 36 & 43 & 44 \\
$\mathrm{Fe}$ & $\underline{\mathrm{Zn}}$ & $\mathrm{Kr}$ & $\underline{\mathrm{Tc}}$ & $\underline{\mathrm{Ru}}$ \\
\hline 45 & 46 & 47 & 49 & 59 \\
$\mathrm{Rh}$ & $\mathrm{Pd}$ & $\mathrm{Ag}$ & $\mathrm{In}$ & $\mathrm{Pr}$ \\
\hline 60 & 61 & 62 & 63 & 64 \\
$\mathrm{Nd}$ & $\mathrm{Pm}$ & $\mathrm{Sm}$ & $\mathrm{Eu}$ & $\mathrm{Gd}$ \\
\hline 65 & 79 & 87 & 88 & 93 \\
$\mathrm{~Tb}$ & $\mathrm{Au}$ & $\underline{\mathrm{Fr}}$ & $\mathrm{Ra}$ & $\underline{\mathrm{Np}}$ \\
\hline
\end{tabular}

The blue shading is for the chemical elements with the odd category in the group of elements $(1,3,5 \ldots 25)$, and green for chemical elements with even category in the same group of chemical elements $(2,4,6 \ldots 24)$. 
Even rank $=(9+24+26+36+44 \ldots+88)=587$;

Odd rank $=(8+15+25+30+43 \ldots+93)=641$;

Even rank code $=587$; Analog even rank code $=785$;

Odd rank code $=641$; Analog odd rank code $=146$;

$(785+146)=$ Discret code $\mathbf{9 3 1}>\mathbf{3}^{\mathbf{2}} \mathbf{3}^{\mathbf{1}} \mathbf{3}^{\mathbf{0}}$.

Even and odd column
\begin{tabular}{|c|c|c|c|c|}
\hline 8 & 9 & 15 & 24 & 25 \\
$\mathrm{O}$ & $\mathrm{F}$ & $\mathrm{P}$ & $\mathrm{Cr}$ & $\mathrm{Mn}$ \\
\hline 26 & 30 & 36 & 43 & 44 \\
$\mathrm{Fe}$ & $\underline{\mathrm{Zn}}$ & $\underline{\mathrm{Kr}}$ & $\underline{\mathrm{Tc}}$ & $\underline{\mathrm{Ru}}$ \\
\hline 45 & 46 & 47 & 49 & 59 \\
$\mathrm{Rh}$ & $\underline{\mathrm{Pd}}$ & $\underline{\mathrm{Ag}}$ & $\underline{\mathrm{In}}$ & $\underline{\mathrm{Pr}}$ \\
\hline 60 & 61 & 62 & 63 & 64 \\
$\underline{\mathrm{Nd}}$ & $\underline{\mathrm{Pm}}$ & $\underline{\mathrm{Sm}}$ & $\underline{\mathrm{Eu}}$ & $\underline{\mathrm{Gd}}$ \\
\hline 65 & 79 & 87 & 88 & 93 \\
$\underline{\mathrm{Tb}}$ & $\underline{\mathrm{Au}}$ & $\underline{\mathrm{Fr}}$ & $\underline{\mathrm{Ra}}$ & $\underline{\mathrm{Np}}$ \\
\hline
\end{tabular}

The blue shading is chemical elements positioned into odd columns $(1,3,5)$, and green for the elements in the even columns $(2,4)$

$$
\begin{aligned}
& \text { Even columns }=(9+30+46+61 \ldots+88)=492 \\
& \text { Odd columns }=(8+26+45+60 \ldots+93)=736
\end{aligned}
$$

Even columns code $=492$; Analog even rank code $=294$;

Odd columns code $=736 ;$ Analog odd rank code $=637$

$$
(294+637)=\text { Discret code } \mathbf{9 3 1}>\mathbf{3}^{\mathbf{2}} \mathbf{3}^{\mathbf{1}} \mathbf{3}^{\mathbf{0}} .
$$

In this example, chemical elements in even and odd columns are connected through their atomic numbers and analogue codes. 


\section{Diagonals}

\begin{tabular}{|c|c|c|c|c|}
\hline 8 & 9 & 15 & 24 & 25 \\
$\underline{0}$ & $\underline{F}$ & $\underline{P}$ & $\underline{\mathrm{Cr}}$ & $\underline{\mathrm{Mn}}$ \\
\hline 26 & 30 & 36 & 43 & 44 \\
$\underline{\mathrm{Fe}}$ & $\underline{\mathrm{Zn}}$ & $\underline{\mathrm{Kr}}$ & $\underline{\mathrm{Tc}}$ & $\underline{\mathrm{Ru}}$ \\
\hline 45 & 46 & 47 & 49 & 59 \\
$\underline{\mathrm{Rh}}$ & $\underline{\mathrm{Pd}}$ & $\underline{\mathrm{Ag}}$ & $\underline{\mathrm{In}}$ & $\underline{\mathrm{Pr}}$ \\
\hline 60 & 61 & 62 & 63 & 64 \\
$\mathrm{Nd}$ & $\mathrm{Pm}$ & $\underline{\mathrm{Sm}}$ & $\underline{\mathrm{Eu}}$ & $\underline{\mathrm{Gd}}$ \\
\hline 65 & 79 & 87 & 88 & 93 \\
$\underline{\mathrm{Tb}}$ & $\underline{\mathrm{Au}}$ & $\underline{\mathrm{Fr}}$ & $\underline{\mathrm{Ra}}$ & $\underline{\mathrm{Np}}$ \\
\hline
\end{tabular}

The blue shading is for the elements out of diagonals, and green for the elements within the diagonals.

Atomic numbers within diagonals $=435 ;$ Analog $\operatorname{code}=534$;

Atomic numbers out of diagonals $=793$; Analog code $=397$;

$$
(534+397)=\text { Discret code } \mathbf{9 3 1}>\mathbf{3}^{\mathbf{2}} \mathbf{3}^{\mathbf{1}} \mathbf{3}^{\mathbf{0}} .
$$

In this example the chemical elements within the diagonals and those out of diagonals are connected through the discrete code 931.

\section{Comment:}

Interpreting as: ListCorrelate $[\{8,30,47,63,93,25,43,7,61,65\},\{9,15,24,26,36$, $44,45,46,49,59,60,62,64,79,87,88\}^{\wedge}$ Conjugate[x], $\left.\{1\}\right]$

\section{Input:}

ListCorrelate $[\{8,30,47,63,93,25,43,7,61,65\}$,

$\left.\{9,15,24,26,36,44,45,46,49,59,60,62,64,79,87,88\}^{x^{*}},\{1\}\right]$ 


\section{Result:}

$$
\begin{aligned}
& \left\{31 \times 3^{2 x^{*}+1} 4^{x^{*}}+8 \times 9^{x^{*}}+2 \times 15^{x^{*}+1}+47 \times 24^{x^{*}}+\right. \\
& 63 \times 26^{x^{*}}+25 \times 44^{x^{*}}+43 \times 45^{x^{*}}+7 \times 46^{x^{*}}+61 \times 49^{x^{*}}+65 \times 59^{x^{*}}, \\
& 5 \times 2^{3 x^{*}+1} 3^{x^{*}+1}+7^{2 x^{*}+1}+5^{x^{*}+2} 9^{x^{*}}+7 \times 4^{x^{*}} 9^{x^{*}+1}+13 \times 5^{x^{*}+1} 12^{x^{*}}+ \\
& 8 \times 15^{x^{*}}+47 \times 26^{x^{*}}+93 \times 44^{x^{*}}+43 \times 46^{x^{*}}+61 \times 59^{x^{*}} \text {, } \\
& 31 \times 3^{2 x^{*}+1} 5^{x^{*}}+3^{x^{*}} 8^{x^{*}+1}+15 \times 2^{x^{*}+1} 13^{x^{*}}+47 \times 36^{x^{*}}+63 \times 44^{x^{*}}+ \\
& 25 \times 46^{x^{*}}+43 \times 49^{x^{*}}+7 \times 59^{x^{*}}+61 \times 60^{x^{*}}+65 \times 62^{x^{*}} \text {, } \\
& 5 \times 6^{2 x^{*}+1}+7 \times 5^{x^{*}} 9^{x^{*}+1}+2^{x^{*}+3} 13^{x^{*}}+47 \times 44^{x^{*}}+93 \times 46^{x^{*}}+ \\
& 25 \times 49^{x^{*}}+43 \times 59^{x^{*}}+7 \times 60^{x^{*}}+61 \times 62^{x^{*}}+65 \times 64^{x^{*}} \text {, } \\
& 2^{2 x^{*}+3} 9^{x^{*}}+15 \times 2^{2 x^{*}+1} 11^{x^{*}}+47 \times 45^{x^{*}}+63 \times 46^{x^{*}}+93 \times 49^{x^{*}}+ \\
& 25 \times 59^{x^{*}}+43 \times 60^{x^{*}}+7 \times 62^{x^{*}}+61 \times 64^{x^{*}}+65 \times 79^{x^{*}}, \\
& 2 \times 3^{2 x^{*}+1} 5^{x^{*}+1}+9 \times 7^{2 x^{*}+1}+2^{2 x^{*}+3} 11^{x^{*}}+5^{x^{*}+2} 12^{x^{*}}+47 \times 46^{x^{*}}+ \\
& 93 \times 59^{x^{*}}+43 \times 62^{x^{*}}+7 \times 64^{x^{*}}+61 \times 79^{x^{*}}+65 \times 87^{x^{*}} \text {, } \\
& 31 \times 3^{x^{*}+1} 20^{x^{*}}+15 \times 2^{x^{*}+1} 23^{x^{*}}+8 \times 45^{x^{*}}+47 \times 49^{x^{*}}+63 \times 59^{x^{*}}+ \\
& 25 \times 62^{x^{*}}+43 \times 64^{x^{*}}+7 \times 79^{x^{*}}+61 \times 87^{x^{*}}+65 \times 88^{x^{*}}, \\
& 65 \times 9^{x^{*}}+7 \times 3^{x^{*}+2} 20^{x^{*}}+2^{x^{*}+3} 23^{x^{*}}+3 \times 2^{x^{*}} 31^{x^{*}+1}+30 \times 49^{x^{*}}+ \\
& 47 \times 59^{x^{*}}+25 \times 64^{x^{*}}+43 \times 79^{x^{*}}+7 \times 87^{x^{*}}+61 \times 88^{x^{*}} \text {, } \\
& 13 \times 3^{x^{*}} 5^{x^{*}+1}+61 \times 9^{x^{*}}+8 \times 49^{x^{*}}+30 \times 59^{x^{*}}+47 \times 60^{x^{*}}+63 \times 62^{x^{*}}+ \\
& 93 \times 64^{x^{*}}+25 \times 79^{x^{*}}+43 \times 87^{x^{*}}+7 \times 88^{x^{*}}, 7 \times 9^{x^{*}}+61 \times 15^{x^{*}}+2^{2 x^{*}+1} 15^{x^{*}+1}+ \\
& 65 \times 24^{x^{*}}+8 \times 59^{x^{*}}+47 \times 62^{x^{*}}+63 \times 64^{x^{*}}+93 \times 79^{x^{*}}+25 \times 87^{x^{*}}+43 \times 88^{x^{*}} \text {, } \\
& 43 \times 9^{x^{*}}+5 \times 2^{x^{*}} 13^{x^{*}+1}+7 \times 15^{x^{*}}+2^{2 x^{*}+3} 15^{x^{*}}+61 \times 24^{x^{*}}+ \\
& 31 \times 3^{x^{*}+1} 29^{x^{*}}+15 \times 2^{x^{*}+1} 31^{x^{*}}+47 \times 64^{x^{*}}+63 \times 79^{x^{*}}+25 \times 88^{x^{*}} \text {, } \\
& 15 \times 2^{6 x^{*}+1}+25 \times 9^{x^{*}}+43 \times 15^{x^{*}}+7 \times 24^{x^{*}}+61 \times 26^{x^{*}}+7 \times 3^{x^{*}+2} 29^{x^{*}}+ \\
& 2^{x^{*}+3} 31^{x^{*}}+65 \times 36^{x^{*}}+47 \times 79^{x^{*}}+93 \times 88^{x^{*}}, 31 \times 3^{2 x^{*}+1}+3^{x^{*}} 5^{x^{*}+2}+8^{2 x^{*}+1}+ \\
& 43 \times 24^{x^{*}}+7 \times 26^{x^{*}}+61 \times 36^{x^{*}}+65 \times 44^{x^{*}}+30 \times 79^{x^{*}}+47 \times 87^{x^{*}}+63 \times 88^{x^{*}} \text {, } \\
& 31 \times 3^{x^{*}+1} 5^{x^{*}}+13 \times 5^{x^{*}+1} 9^{x^{*}}+7 \times 9^{x^{*}+1}+25 \times 24^{x^{*}}+43 \times 26^{x^{*}}+ \\
& 10 \times 3^{x^{*}+1} 29^{x^{*}}+7 \times 36^{x^{*}}+61 \times 44^{x^{*}}+8 \times 79^{x^{*}}+47 \times 88^{x^{*}} \text {, } \\
& 7 \times 3^{x^{*}+2} 5^{x^{*}}+31 \times 3^{x^{*}+1} 8^{x^{*}}+47 \times 9^{x^{*}}+15 \times 2^{3 x^{*}+1} 11^{x^{*}}+25 \times 26^{x^{*}}+ \\
& 43 \times 36^{x^{*}}+7 \times 44^{x^{*}}+61 \times 45^{x^{*}}+65 \times 46^{x^{*}}+8 \times 87^{x^{*}} \text {, } \\
& 10 \times 3^{2 x^{*}+1}+7 \times 3^{x^{*}+2} 8^{x^{*}}+8^{x^{*}+1} 11^{x^{*}}+47 \times 15^{x^{*}}+93 \times 26^{x^{*}}+ \\
& \left.25 \times 36^{x^{*}}+43 \times 44^{x^{*}}+7 \times 45^{x^{*}}+61 \times 46^{x^{*}}+65 \times 49^{x^{*}}\right\}
\end{aligned}
$$




\section{Plot:}
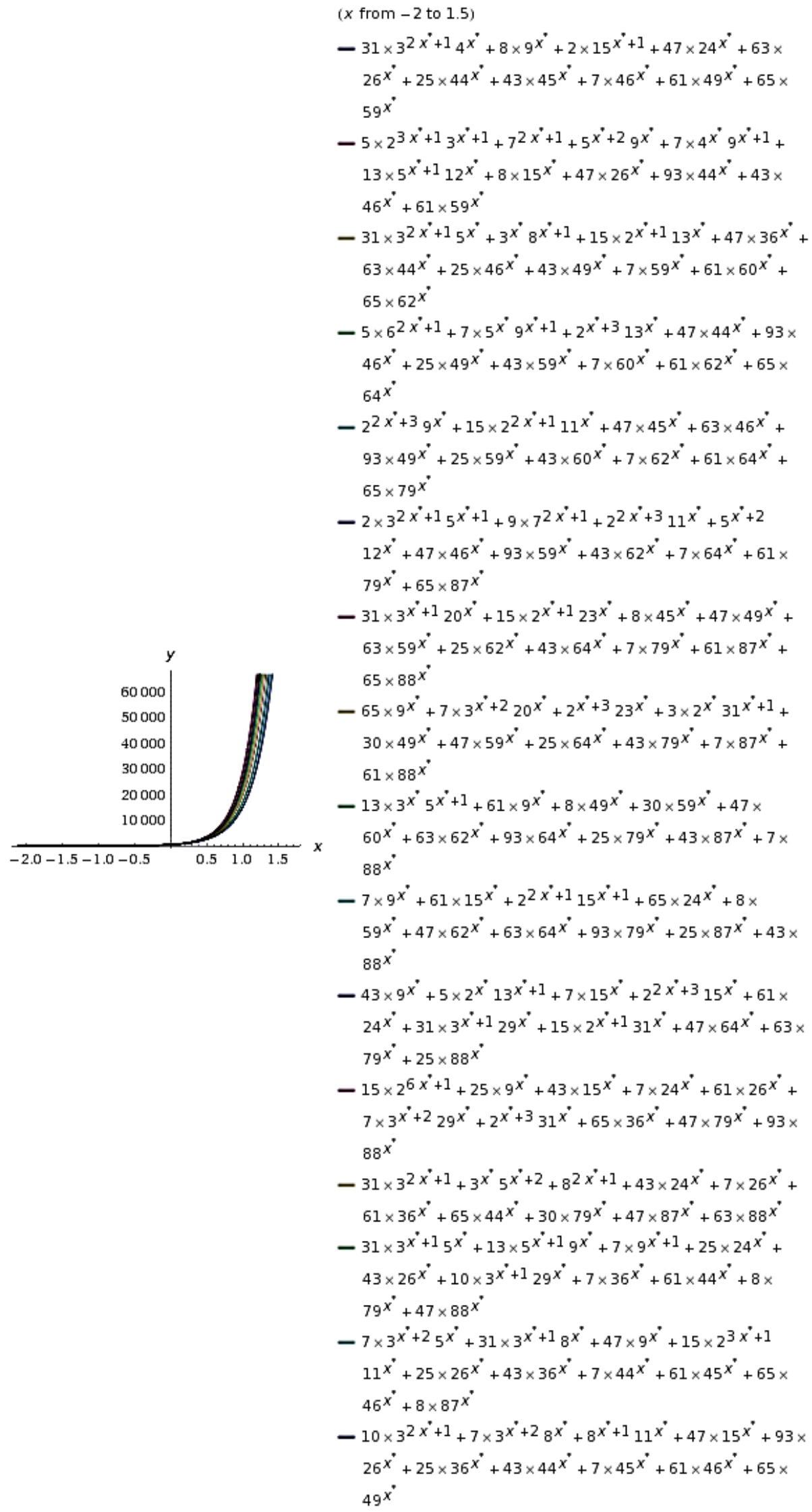
Odd inner numbers

\begin{tabular}{|c|c|c|c|c|}
\hline 8 & 9 & 15 & 24 & 25 \\
$\underline{O}$ & $\underline{F}$ & $\underline{P}$ & $\underline{\mathrm{Cr}}$ & $\underline{\mathrm{Mn}}$ \\
\hline 26 & 30 & 36 & 43 & 44 \\
$\underline{\mathrm{e}}$ & $\underline{\mathrm{Zn}}$ & $\underline{\mathrm{Kr}}$ & $\underline{\mathrm{Tc}}$ & $\underline{\mathrm{Ru}}$ \\
\hline 45 & 46 & 47 & 49 & 59 \\
$\underline{\mathrm{Rh}}$ & $\underline{\mathrm{Pd}}$ & $\underline{\mathrm{Ag}}$ & $\underline{\mathrm{In}}$ & $\underline{\mathrm{Pr}}$ \\
\hline 60 & 61 & 62 & 63 & 64 \\
$\underline{\mathrm{Nd}}$ & $\underline{\mathrm{Pm}}$ & $\underline{\mathrm{Sm}}$ & $\underline{\mathrm{Eu}}$ & $\underline{\mathrm{Gd}}$ \\
\hline 65 & 79 & 87 & 88 & 93 \\
$\underline{\mathrm{Tb}}$ & $\underline{\mathrm{Au}}$ & $\underline{\mathrm{Fr}}$ & $\underline{\mathrm{Ra}}$ & $\underline{\mathrm{Np}}$ \\
\hline
\end{tabular}

Odd inner numbers $=(43+47+49+61+63)=263 ;$ Analog code $=362$;

Other numbers in square $=(8+9+15+24+25+26+30+36+44+45+46+59+60+62+$ $+64+65+79+87+88+93)=965 ;$ Analog code $=569$;

$$
(362+569)=931
$$

\section{Columns}

\begin{tabular}{|c|c|c|c|c|}
\hline 8 & 9 & 15 & 24 & 25 \\
$\underline{\mathrm{O}}$ & $\underline{\mathrm{F}}$ & $\underline{\mathrm{P}}$ & $\underline{\mathrm{Cr}}$ & $\underline{\mathrm{Mn}}$ \\
\hline 26 & 30 & $\mathbf{3 6}$ & 43 & 44 \\
$\mathrm{Fe}$ & $\underline{\mathrm{Zn}}$ & $\underline{\mathrm{Kr}}$ & $\underline{\mathrm{Tc}}$ & $\underline{\mathrm{Ru}}$ \\
\hline 45 & 46 & 47 & 49 & 59 \\
$\mathrm{Rh}$ & $\underline{\mathrm{Pd}}$ & $\underline{\mathrm{Ag}}$ & $\underline{\mathrm{In}}$ & $\underline{\mathrm{Pr}}$ \\
\hline 60 & 61 & 62 & 63 & 64 \\
$\mathrm{Nd}$ & $\underline{\mathrm{Pm}}$ & $\underline{\mathrm{Sm}}$ & $\underline{\mathrm{Eu}}$ & $\underline{\mathrm{Gd}}$ \\
\hline 65 & 79 & $\mathbf{8 7}$ & $\mathbf{8 8}$ & 93 \\
$\underline{\mathrm{Tb}}$ & $\underline{\mathrm{Au}}$ & $\underline{\mathrm{Fr}}$ & $\underline{\mathrm{Ra}}$ & $\underline{\mathrm{Np}}$ \\
\hline
\end{tabular}

Number in 4. and 5. columns $=(24+25+43+44+49+59+63+64+88+93)=552$; Analog code $=255$;

Numbers in 1 st, 2 nd, and 3 rd columns $=(8+9+15+26+30+36+45+46+47+60+61++62+$ $65+79+87)=676 ;$ Analog code $=676$;

$$
(255+676)=931
$$




\section{Odd numbers in odd columns}

\begin{tabular}{|c|c|c|c|c|}
\hline $\mathbf{8}$ & 9 & $\mathbf{1 5}$ & $\mathbf{2 4}$ & $\mathbf{2 5}$ \\
$\underline{\mathrm{O}}$ & $\underline{\mathrm{F}}$ & $\underline{\mathrm{P}}$ & $\underline{\mathrm{Cr}}$ & $\mathrm{Mn}$ \\
\hline $\mathbf{2 6}$ & $\mathbf{3 0}$ & $\mathbf{3 6}$ & $\mathbf{4 3}$ & $\mathbf{4 4}$ \\
$\underline{\mathrm{e}}$ & $\underline{\mathrm{Zn}}$ & $\underline{\mathrm{Kr}}$ & $\underline{\mathrm{Tc}}$ & $\underline{\mathrm{Ru}}$ \\
\hline 45 & 46 & 47 & 49 & 59 \\
$\mathrm{Rh}$ & $\underline{\mathrm{Pd}}$ & $\underline{\mathrm{Ag}}$ & $\underline{\mathrm{In}}$ & $\underline{\mathrm{Pr}}$ \\
\hline $\mathbf{6 0}$ & $\mathbf{6 1}$ & $\mathbf{6 2}$ & $\mathbf{6 3}$ & $\mathbf{6 4}$ \\
$\underline{\mathrm{Nd}}$ & $\underline{\mathrm{Pm}}$ & $\underline{\mathrm{Sm}}$ & $\underline{\mathrm{Eu}}$ & $\underline{\mathrm{Gd}}$ \\
\hline$\underline{65}$ & $\mathbf{7 9}$ & $\mathbf{8 7}$ & $\mathbf{8 8}$ & $\mathbf{9 3}$ \\
$\underline{\mathrm{Tb}}$ & $\underline{\mathrm{Au}}$ & $\underline{\mathrm{Fr}}$ & $\underline{\mathrm{Ra}}$ & $\underline{\mathrm{Np}}$ \\
\hline
\end{tabular}

Odd numbers in odd columns $=(15+25+45+47+59+65+87+93)=436$;

Analog code $=634$

Other numbers $=(8+9+24+26+30+36+43+44+46+49+60+61+62+$

$+63+64+79+88)=792$;

Analog code $=297$

$(634+297)=931$

Odd numbers in odd rows

\begin{tabular}{|c|c|c|c|c|}
\hline 8 & 9 & 15 & 24 & 25 \\
$\underline{\mathrm{O}}$ & $\underline{\mathrm{F}}$ & $\underline{\mathrm{P}}$ & $\underline{\mathrm{Cr}}$ & $\underline{\mathrm{Mn}}$ \\
\hline 26 & $\mathbf{3 0}$ & $\mathbf{3 6}$ & $\mathbf{4 3}$ & $\mathbf{4 4}$ \\
$\mathrm{Fe}$ & $\underline{\mathrm{Zn}}$ & $\underline{\mathrm{Kr}}$ & $\underline{\mathrm{Tc}}$ & $\underline{\mathrm{Ru}}$ \\
\hline 45 & 46 & 47 & 49 & 59 \\
$\underline{\mathrm{Rh}}$ & $\underline{\mathrm{Pd}}$ & $\underline{\mathrm{Ag}}$ & $\underline{\mathrm{In}}$ & $\underline{\mathrm{Pr}}$ \\
\hline 60 & 61 & $\mathbf{6 2}$ & $\mathbf{6 3}$ & $\mathbf{6 4}$ \\
$\underline{\mathrm{Nd}}$ & $\underline{\mathrm{Pm}}$ & $\underline{\mathrm{Sm}}$ & $\underline{\mathrm{Eu}}$ & $\underline{\mathrm{Gd}}$ \\
\hline 65 & 79 & $\mathbf{8 7}$ & $\mathbf{8 8}$ & 93 \\
$\mathrm{~Tb}$ & $\underline{\mathrm{Au}}$ & $\underline{\mathrm{Fr}}$ & $\underline{\mathrm{Ra}}$ & $\underline{\mathrm{Np}}$ \\
\hline
\end{tabular}

Odd numbers in odd rows $=(9+15+25+45+47+49+59+65+79+87+93)=573$; Analog code $=375$;

Other numbers in square $=(8+24+26+30+36+43+44+46+60+61+62+63+64+88)=655$;

Analog code $=556$;

$$
(375+556)=931 ;
$$


Odd numbers in diagonals

\begin{tabular}{|c|c|c|c|c|}
\hline 8 & 9 & 15 & 24 & 25 \\
$\underline{\mathrm{O}}$ & $\underline{\mathrm{F}}$ & $\underline{\mathrm{P}}$ & $\underline{\mathrm{Cr}}$ & $\underline{\mathrm{Mn}}$ \\
\hline 26 & $\mathbf{3 0}$ & $\mathbf{3 6}$ & 43 & 44 \\
$\mathrm{Fe}$ & $\underline{\mathrm{Zn}}$ & $\underline{\mathrm{Kr}}$ & $\underline{\mathrm{Tc}}$ & $\underline{\mathrm{Ru}}$ \\
\hline 45 & 46 & 47 & 49 & $\mathbf{5 9}$ \\
$\underline{\mathrm{Rh}}$ & $\underline{\mathrm{Pd}}$ & $\underline{\mathrm{Aq}}$ & $\underline{\mathrm{In}}$ & $\underline{\mathrm{Pr}}$ \\
\hline 60 & 61 & 62 & 63 & 64 \\
$\underline{\mathrm{Nd}}$ & $\underline{\mathrm{Pm}}$ & $\underline{\mathrm{Sm}}$ & $\underline{\mathrm{Eu}}$ & $\underline{\mathrm{Gd}}$ \\
\hline 65 & 79 & $\mathbf{8 7}$ & $\mathbf{8 8}$ & 93 \\
$\underline{\mathrm{Tb}}$ & $\underline{\mathrm{Au}}$ & $\underline{\mathrm{Fr}}$ & $\underline{\mathrm{Ra}}$ & $\underline{\mathrm{Np}}$ \\
\hline
\end{tabular}

Odd numbers in diagonals $=(25+43+47+61+63+65+93)=397$

Analog code $=793$;

Other numbers in square $=(8+9+15+24+26+30+36+44+45+46+49+59+60+62+$ $+64+79+87+88)=831$;

Analog code $=138$

$(793+138)=931$

Odd numbers in 4. and 5. Columns

\begin{tabular}{|c|c|c|c|c|}
\hline 8 & 9 & 15 & 24 & 25 \\
$\underline{\mathrm{O}}$ & $\underline{\mathrm{F}}$ & $\underline{\mathrm{P}}$ & $\underline{\mathrm{Cr}}$ & $\underline{\mathrm{Mn}}$ \\
\hline 26 & $\mathbf{3 0}$ & $\mathbf{3 6}$ & 43 & 44 \\
$\mathrm{Fe}$ & $\underline{\mathrm{Zn}}$ & $\underline{\mathrm{Kr}}$ & $\underline{\mathrm{Tc}}$ & $\underline{\mathrm{Ru}}$ \\
\hline$\underline{45}$ & 46 & 47 & 49 & 59 \\
$\mathrm{Rh}$ & $\underline{\mathrm{Pd}}$ & $\underline{\mathrm{Ag}}$ & $\underline{\mathrm{In}}$ & $\underline{\mathrm{Pr}}$ \\
\hline 60 & 61 & 62 & 63 & 64 \\
$\underline{\mathrm{Nd}}$ & $\underline{\mathrm{Pm}}$ & $\underline{\mathrm{Sm}}$ & $\underline{\mathrm{Eu}}$ & $\underline{\mathrm{Gd}}$ \\
\hline 65 & $\mathbf{7 9}$ & $\mathbf{8 7}$ & $\mathbf{8 8}$ & 93 \\
$\underline{\mathrm{Tb}}$ & $\underline{\mathrm{Au}}$ & $\underline{\mathrm{Fr}}$ & $\underline{\mathrm{Ra}}$ & $\underline{\mathrm{Np}}$ \\
\hline
\end{tabular}

Odd numbers in 4 . and 5 . columns $=(25+43+49+59+63+93)=332$;

Analog code $=233$;

Other numbers in square $=(8+9+15+24+26+30+36+44+45+46+47+60+61+62++64+$ $65+79+87+88)=896 ;$ Analog code $=698$;

$$
(233+698)=931 \text {; }
$$




\section{Right and left numbers}

The square that we are talking about is consisted of 25 different numbers. Some of those are made of one number and some of two. Some numbers are on the right, and some numbers are on the left side. Now lets analyze those numbers; Numbers on the right side are: 8, 9, 5, 4, $5,6,0,6,3,4,5,6,7,9,9,0,1,2,3,4,5,9,7,8$ and 3 . Total number is 128 , and analogue code is 821 . Number on the left side are: $0,0,1,2,2,2,3,3,4,4,4,4,4,4,5,6,6,6,6,6,6$, $7,8,8$, and 9.The total of numbers is 110 , and analogue is 011 .

Now we are going to summarize the totals and analogue codes.

$$
(128+821)+(110+011)=(139+931)
$$

As we can see, numbers on the right and the left side are coded in the number 931, and his analogue code.

\section{CONNECTION OF CORRESPONDING NUMBERS}

Some of the secrets of this square we can decode when we connect numbers with the first numbers of squares.

(Rank of the square and Number in the square) $=$ Connecting number;

\section{Example 1}

$(15$ and 59$)=1559$

$(21$ and 65$)=2165$

$$
(1559+2165)=3724=(931+931+931+931)
$$

\section{Example 2}

(Rank of the square and Number in the square) = Connecting number;

$$
\begin{gathered}
(16 \text { and } 60)=1660 ; \\
(20 \text { and } 64)=2064 ; \\
(1660+2064)=3724=(931+931+931+931) \\
\text { Example } 3
\end{gathered}
$$

(Rank of the square and Number in the square) = Connecting number;

$(17$ and 61$)=1761$

$(19$ and 63$)=1963$; 


$$
(1761+1963)=3724=(931+931+931+931)
$$

\section{Example 4}

$($ Rank of the square and Number in the square $)=$ Connecting number;

$$
\begin{aligned}
& (18 \text { and } 62)=1862 ; \\
& 1862=(931+931) ;
\end{aligned}
$$

Example 5

$\begin{array}{cccc}\begin{array}{c}\text { Rank of } \\ \text { the square }\end{array} & \begin{array}{c}\text { Atomic } \\ \text { number }\end{array} & \Rightarrow & \begin{array}{c}\text { Connecting } \\ \text { numbers }\end{array} \\ 17 & 61 & 7 & 1761 \\ 18 & 62 & \Rightarrow & 1862 \\ 19 & 63 & \Rightarrow & 1963 \\ \text { Total } & & & 5586\end{array}$

$$
5586=(931+931+931+931+931+931)
$$

\section{Example 6}

$\begin{array}{cccc}\begin{array}{c}\text { Rank of } \\ \text { the square }\end{array} & \text { Atomic } & & \begin{array}{c}\text { Connecting } \\ \text { numbers }\end{array} \\ 16 & 60 & \Rightarrow & 1660 \\ 18 & 62 & \Rightarrow & 1862 \\ 20 & 64 & \Rightarrow & 2064 \\ \text { Svega } & & & 5586\end{array}$

$$
5586=(931+931+931+931+931+931)
$$

\section{Example 7}

$\begin{array}{cccc}\begin{array}{c}\text { Rank of } \\ \text { the square }\end{array} & \begin{array}{c}\text { Atomic } \\ \text { number }\end{array} & & \begin{array}{c}\text { Connecting } \\ \text { numbers }\end{array} \\ 16 & 60 & \rightarrow & 1660 \\ 17 & 61 & \Rightarrow & 1761 \\ 21 & 65 & \Rightarrow & 2165 \\ \text { Svega } & & & 5586\end{array}$

$$
5586=(931+931+931+931+931+931)
$$

etc. 


\section{Example 1}

Chemical element Br; Atomic number 35;

$$
\mathrm{R}=35
$$

$$
\begin{gathered}
\{[\mathrm{S} 7(35) \times 19]-[\mathrm{S} 19(35) \times 7]+(7 \times 19)\}=(7 \times 19 \times 7) \\
\mathrm{S} 7(35)=(29+30+31+32+33+34+35)=224
\end{gathered}
$$

$\mathrm{S} 19(35)=(17+18+19+20+21+22+23+24+25+26+27+28+29+30+31+32+33+34+35)=494$

$$
(224 \times 19)-(494 \times 7)+(7 \times 19)=(7 \times 19 \times 7)=931
$$

\section{Example 2}

Chemical element Ca; Atomic number 20.

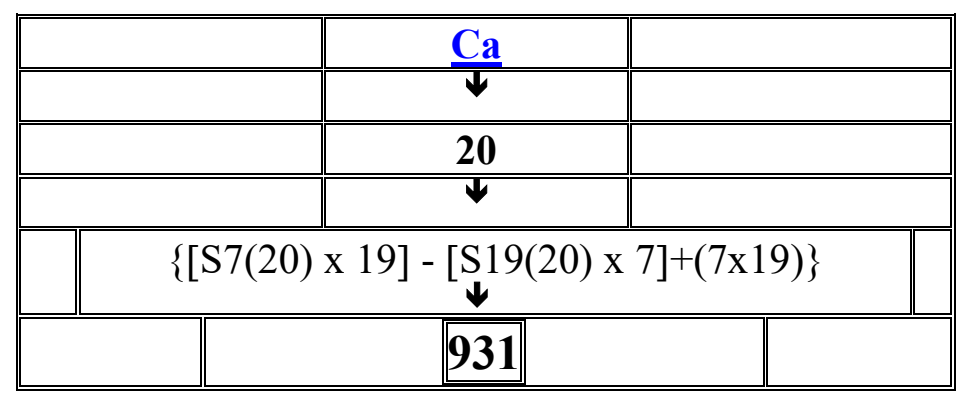

\section{Example 3}

Chemical element $\underline{\mathbf{S r}}$; Atomic number 38.

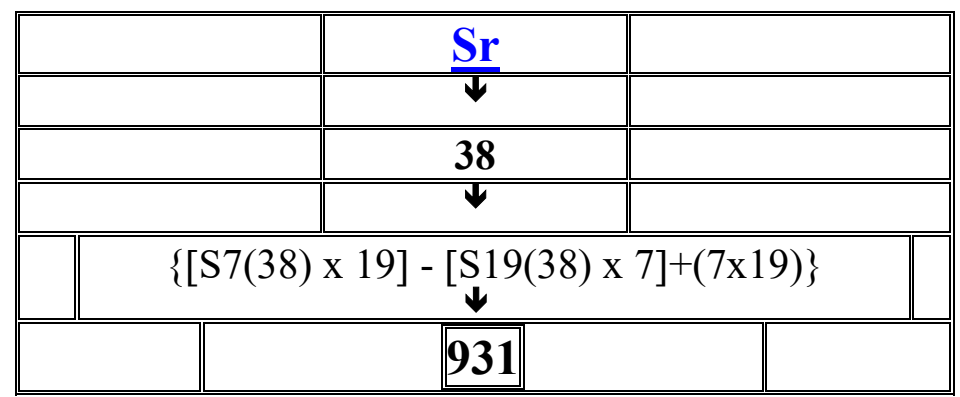




\section{Example 4}

Chemical element $\underline{\mathbf{I}}$; Atomic number 53.

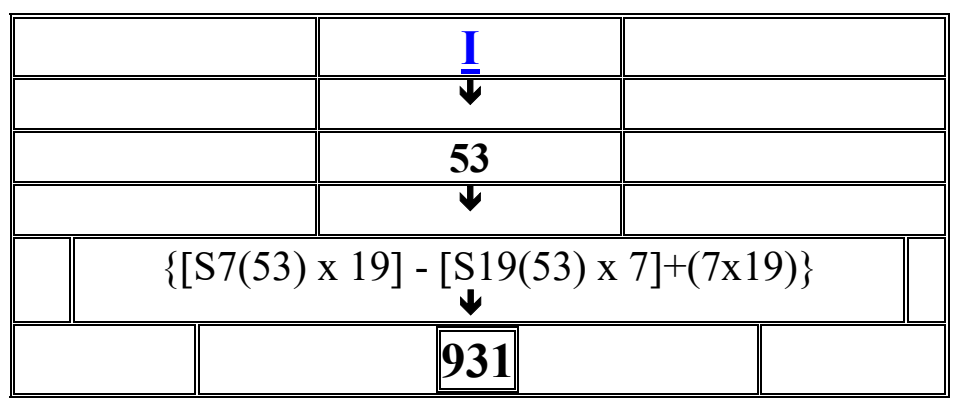

etc.

The mathematical balance in groups of chemical elements from $\mathrm{X}$ to $\mathrm{Y}$ on exsist with help codes $19 \mathrm{i} 7$. Output of these codes is code $\mathbf{9 3 1}$.

In those examples, we translated the physical and chemical parameters from the language of chemistry into the digital language of programmatic, cybernetic and information principles. This we did by using the adequate mathematical algorithms. By using chemicalinformation procedures, we calculated the numerical value for the information content of periodic table.

What we got this way is the digital picture of the phenomenon of chemistry. These digital pictures reveal to us a whole new dimension of this science. They reveal to us that the biochemical process is strictly conditioned and determined by programmatic, cybernetic and information principles.

The digital language of chemistry has a countless number of codes and analogue codes, as well as other information content. These pictures enable us to realize the very essence of functioning of biochemical processes. Here are some examples:

\section{Code 931 in atoms}

The micro structure of atoms are 9 parts. In first and second parts there are protons and neutrons. They are in central part of the atom. Threth, fourth, fiveth, sixth, seventh, eighth and nineth parts are out of central part of atoms. In this part there are the electrons. Arithmetical expression for this 9 parts is number 9 .

The construction of atoms does 3 sequences: protons, elektrons and neutrons.

Arithmetical expression for one atoms is - number 1 .

Numbers: 9,3 and 1 we will connect in number 931:

$$
9,3,1 \Rightarrow \mathbf{9 3 1}
$$




\section{We can see this relation in following table:}

\begin{tabular}{||c||c||c||}
\hline $\begin{array}{c}\text { Number of parts } \\
\text { in atom }\end{array}$ & $\begin{array}{c}\text { Number of principal } \\
\text { sequences in atom }\end{array}$ & $\begin{array}{c}\text { Arithmetical } \\
\text { expression for } \\
\text { one atom }\end{array}$ \\
\hline$\downarrow$ & $\downarrow$ & $\downarrow$ \\
\hline \hline 9 & 3 & 1 \\
\hline \hline $\boldsymbol{y}$ & $\mathbf{9 3 1}$ & $\mathbf{K}$ \\
\hline \hline
\end{tabular}

\section{Periodic system Table}

In the Periodic system Table there are 9 groups of the chemical elements. Also, in this Table these are 3 pairs of the periods. First (1) period has one pairs the elements. Output of those numbers is code 931 :

\begin{tabular}{|c||c|c|}
\hline $\begin{array}{c}\text { Groups of } \\
\text { the } \\
\text { chemical } \\
\text { elements }\end{array}$ & $\begin{array}{c}\text { The pairs of } \\
\text { the periods }\end{array}$ & $\begin{array}{c}\text { First } \\
\text { period has } \\
\text { one pairs } \\
\text { elements }\end{array}$ \\
\hline$\downarrow$ & $\boldsymbol{V}$ & $\boldsymbol{}$ \\
\hline \hline 9 & 3 & 1 \\
\hline \hline $\mathbf{Y}$ & $\boldsymbol{\mathbf { K }}$ \\
\hline & $\mathbf{9 3 1}$ & \\
\hline
\end{tabular}

Molecule $\mathrm{H}_{2} \mathrm{O}$

\begin{tabular}{|c||c||c|}
\hline $\begin{array}{c}\text { Atomic } \\
\text { numbers } \\
\mathrm{H} \text { and } \mathrm{O} \\
(1+8)\end{array}$ & $\begin{array}{c}\text { Number of atoms in } \\
\text { molecule of the water }\end{array}$ & $\begin{array}{c}\text { Atomic } \\
\text { number } \\
\text { (H) }\end{array}$ \\
\hline $\boldsymbol{\downarrow}$ & $\boldsymbol{\downarrow}$ & $\boldsymbol{\downarrow}$ \\
\hline 9 & 3 & 1 \\
\hline $\mathbf{Y}$ & $\boldsymbol{\mathbf { M }}$ & $\boldsymbol{K}$ \\
\hline & $\mathbf{9 3 1}$ & \\
\hline
\end{tabular}

Wavelenghth

Wavelenghth start from 380 to 780 .

Groups of all wavelenghth $=(380+381+382 \ldots+780)=224390$;

$$
\begin{gathered}
224390=(197 * X 1)+(931 * X 2) \\
X 1=19 ; X 2=237 ; \\
224390=(197 * X 3)+(931 * X 4)
\end{gathered}
$$




$$
\begin{gathered}
X 3=950 ; X 2=40 \\
(X 3-X 1)=931:
\end{gathered}
$$

Avogard's number

$$
\begin{aligned}
& \text { Avogard's number }=6,02214 \times 10^{23} \\
& 602214=[(197+931)+(197+931)+931 \times Y)+(931+197)+(931+197)] \\
& \mathrm{Y}=646 \text {; }
\end{aligned}
$$

etc.

There are discrete codes that can show us one radical new dimension of the biochemical processes functioning. And in that dimension we can find an explanation for the given empirical reality.

\section{CONCLUSIONS}

Digital image of the chemistry is an image in which biochemistry is converted to mathematics. Actually, we convert it to numbers. As soon as we convert to numbers we'll get digital image of the chemistry. In that digital image, there is some very significant scientific information. Those are information in which is given explanation of that reality. That digital image of the chemistry will enable current science to significantly advance it's scientificresearch work and to develop top digital technologies in this science in a very short time.

\section{References}

[1] L. Kurić, International Letters of Chemistry, Physics and Astronomy 10 (2014) 62-73.

[2] L. Kurić, J. Comput Sci Biol 2 (2009) 101-116.

[3] L. Kurić, International Letters of Chemistry, Physics and Astronomy 13(1) (2014) 42-53

[4] L. Kurić, Journal de la Societe de statistique de Paris 127(2) (1986).

[5] L. Kurić, GJMR 10(1) (2010) 15.

[6] L. Kurić, Advances and Applications in Bioinformatics and Chemistry (2010) 45-58.

[7] L. Kurić, GJMR 1(1) (2010) 15.

[8] L. Kurić, International Journal of Computer Technology and Application 2(2) (2011) 216-241. 
[9] L. Kurić, International Journal of Computer Technology and Application 2(2) (2011) 258-273.

[10] L. Kurić, Journal of Chemical Enginerring and Material Science 2(5) (2011).

[11] L. Kurić, International Letters of Chemistry, Physics and Astronomy 11(3) (2014) 202-213.

[12] L. Kurić, International Letters of Chemistry, Physics and Astronomy 12 (2014) 31-50.

[13] L. Kurić, International Letters of Chemistry, Physics and Astronomy 13(1) (2014) 11-20.

[14] L. Kurić, International Letters of Chemistry, Physics and Astronomy 12 (2014) 31-50

[15] L. Kurić, International Letters of Chemistry, Physics and Astronomy 13(2) (2014) 119-134.

[16] L. Kurić, International Letters of Chemistry, Physics and Astronomy 13(2) (2014) 160-173.

[17] L. Kurić, International Letters of Chemistry, Physics and Astronomy 13(2) (2014) 174-190. 